\title{
Study on the Vibration Reduction of the Double Pistons Hydraulic Engine Mount
}

\author{
You-Gang Tang \\ Professor, Dr., Department of Naval Architecture and Marine Engineering, School of Architecture Engineering, Tianjin \\ University, Tianjin, 300072, P.R.C. \\ Hong-Yu Zheng \\ Graduate Students, Department of Naval Architecture and Marine Engineering, School of Architecture Engineering, \\ Tianjin University, Tianjin, 300072, P.R.C. \\ Jia-Yang Gu \\ Graduate Students, Department of Naval Architecture and Marine Engineering, School of Architecture Engineering, \\ Tianjin University, Tianjin, 300072, P.R.C. \\ Da-Ning Jiang \\ Graduate Students, Department of Naval Architecture and Marine Engineering, School of Architecture Engineering, \\ Tianjin University, Tianjin, 300072, P.R.C.
}

Follow this and additional works at: https://jmstt.ntou.edu.tw/journal

Part of the Engineering Commons

\section{Recommended Citation}

Tang, You-Gang; Zheng, Hong-Yu; Gu, Jia-Yang; and Jiang, Da-Ning (2003) "Study on the Vibration Reduction of the Double Pistons Hydraulic Engine Mount," Journal of Marine Science and Technology. Vol. 11: Iss. 1, Article 6. DOI: $10.51400 / 2709-6998.2278$

Available at: https://jmstt.ntou.edu.tw/journal/vol11/iss1/6

This Research Article is brought to you for free and open access by Journal of Marine Science and Technology. It has been accepted for inclusion in Journal of Marine Science and Technology by an authorized editor of Journal of Marine Science and Technology. 


\title{
STUDY ON THE VIBRATION REDUCTION OF THE DOUBLE PISTONS HYDRAULIC ENGINE MOUNT
}

\author{
You-Gang Tang*, Hong-Yu Zheng**, Jia-Yang Gu**, and Da-Ning Jiang**
}

Key words: hydraulic engine mount, vibration reduction, dynamic stiffness, transfer function.

\begin{abstract}
A novelty style metal hydraulic engine mount (HEM) is developed. The HEM designed in this paper consists of hydraulic cylinder, bar, two pistons and the spring on the bottom. The mechanical model and the dynamic equations are set up by considering kinematics and continuous of fluid, and the analytical method for dynamic behavior including dynamic stiffness and transfer function of HEM are suggested herein. The dynamic characteristics of HEM are investigated. It is shown that the HEM proposed herein can be employed in the practical engineering for vibration reduction.
\end{abstract}

\section{INTRODUCTION}

The rubber mount is often adopted to reduce vibration and noise of cars, but the rubber dampers have poorer performance of vibration reduction in lower frequencies. In order to improve the vibration and noise of cars in broad frequencies, the hydraulic rubber mount for engine of a car was presented in 1984, Waliace summarized the constructions of hydraulic mounts and mechanism of vibration reduction [8]. In general, there are 4 type of HEM [2-4, 6]: HEM with simple orifice; HEM with inertia track; HEM with orifice and decouple; HEM with inertia track and de-couple. The dynamic performances of HEM with simple orifice depend on the area of orifice and rubber element. The optimization theory and analytical model of HEM were presented to minize transfer function of vibration [3]. Gau and Jeffry [2] carried out the theory and experiment

Paper Submitted 08/07/02, Accepted 04/09/03. Author for Correspondence: You-Gang Tang.

*Professor, Dr., Department of Naval Architecture and Marine Engineering, School of Architecture Engineering, Tianjin University, Tianjin, 300072, P.R.C.

**Graduate Students, Department of Naval Architecture and Marine Engineering, School of Architecture Engineering, Tianjin University, Tianjin, 300072, P.R.C. study of the HEM with orifice and de-couple, it is shown that the HEM has high damping in $5-15 \mathrm{~Hz}$ frequency region, and low damping in $100-300 \mathrm{~Hz}$ frequency region, whose are suitable for vibration reduction of cars. The HEM with inertia track comprises the primary rubber element and the inertia track (long tube), thus it can provide higher damping than HEM with orifice, Kim and Singh studied the behaviors of reduction vibration by using the model of engine-mount system, and the results of calculation are identical with the experiment datum [4]. The behavior of vibration reduction of HEM with inertia track and de-couple were investigated with two freedom model and considering the piecewise liner characteristics of de-couple [7]. The HEM mentioned in References [2-4, 6-8] can be called as the Rubber Fluid Absorbers (RFA), which are not suitable for vibration reduction of heavy vehicles. The HEM with metal cylinder and single piston (MCSP) was designed and used in the ambulance stretcher system. The MCSP is composed of metal cylinder and one piston with orifice, when a piston moves up and down, the fluid flows back and forth and thus causes the damping effect [5]. There are some weaknesses in the behaviors both impulse and fatigue of RFA and MCSP. So, it is important to develop novelty HEM for the vibration reduction of heavy machineries and heavy vehicles. The paper improved the HEM based on the construction of RFA and MCSP. The metal cylinder replaces the primary rubber element, the disc with orifice is fixed inside the cylinder, and the two pistons are linked by bar. When the two pistons move up and down with bar simultaneously, it forces the fluid to flow through the orifice, which produces higher damping than MCSP. The HEM developed in this paper can be employed in the vibration reduction of heavy vehicles.

\section{ANALYTICAL MODEL AND MOTION EQUATIONS OF THE HEM}

The Fig. 1 shows the HEM with orifice and two pistons. The HEM is composed of two pistons, bar, 
disc with orifice and spring on the bottom. The upper piston and lower piston are linked by bar, and they move with bar simultaneously. Only if the vertical displacement is considered, HEM can be described by the model in Fig. 2.

As a engine moves down, the two pistons move down with bar simultaneously, and the upper chamber is compressed, which forces the fluid to flow through the orifice into the lower chamber, it causes the inertia force and the damping of fluid inside the orifice, and the transfer function of vibration will be reduced by making use of these two forces.

In Fig. 2, $k_{s}$ and $c_{s}$ are the stiffness coefficient and damping coefficient of the bottom spring, respectively; $m_{1}$ is the mass including the two pistons and the bar; $m_{0}$ is the mass on the top of mount; $x$ expresses the inputting deflection; $F$ is the resultant of forces acting on the upper piston. The volume stiffness $k\left(\mathrm{~N} / \mathrm{m}^{5}\right)$ of fluid is defined as the ratio of $\Delta p$ to $\Delta v$ inside the upper chamber, i.e. $k=\Delta p / \Delta v[6,8], \Delta p$ denotes the pressure change, and $\Delta v$ is the volume change inside the upper chamber. $k$ indicates the pressure change induced by unit volume change of upper chamber. $\Delta p$ and $\Delta v$ are induced by pistons motion.

The fluid pressure of upper chamber is defined as $p ; A_{0}$ represents the cross-section area of piston (except the area of bar) and $A_{1}$ is the total area of orifice in the disc; $y$ is the vertical flow of fluid inside the orifice.

Based on the continuous condition of fluid, the change of fluid volume inside the upper chamber should equalize the fluid volume flowing through the orifice i.e.

$$
A_{0} x=A_{1} y
$$

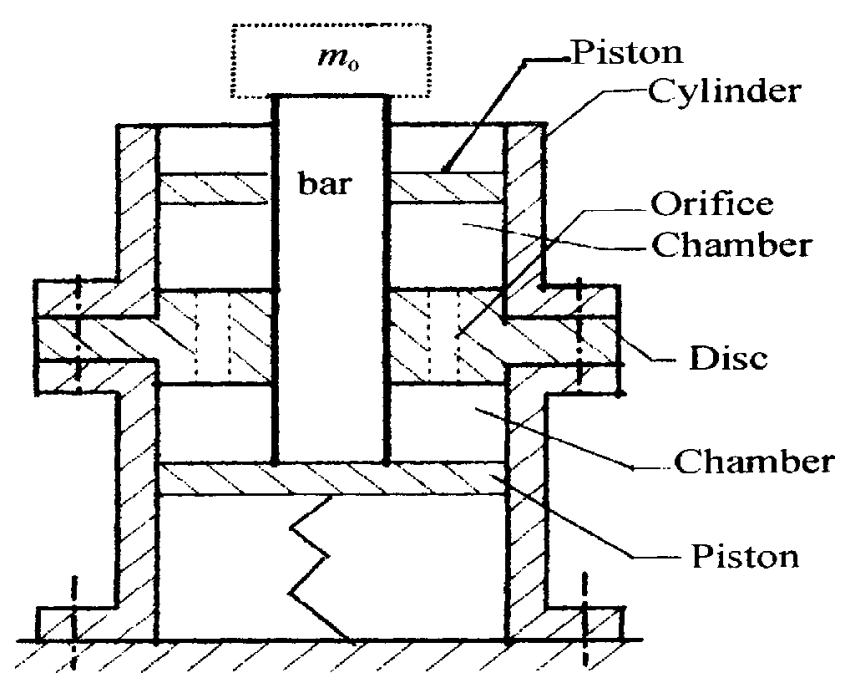

Fig. 1. Hydraulic double-pistons mount. where, $A_{0}$ is the volume change of fluid inside the upper chamber; $A_{1} y$ indicates the fluid volume flowing through the orifice.

It is assumed that the fluid pressure $p$ inside upper chamber is uniform, so the fluid pressure can be given as following

$$
p=\left(A_{1} y\right) k
$$

the vibration equation of the construction can be obtained based on the equilibrium of force,

$$
\left(m_{0}+m_{1}\right) \ddot{x}+c_{s} \dot{x}+k_{s} x+A_{0} p=F
$$

where, $A_{0} p$ is the hydraulic force acting on the piston.

The fluid inside the orifice bears the pressure forces $A_{1} p$, it can be simplified into the oscillator caused by the acceleration of fluid motion through the orifice, and the dynamic equation of the oscillator can be described as following

$$
m_{f} \ddot{y}+c_{f} \dot{y}=A_{1} p
$$

where, $m_{f}$ is the total mass of fluid inside orifice, $m_{f}=$ $A_{1} h \rho, h$ is the height of orifice; $\rho$ denotes the density of fluid; $c_{f}$ expresses the damping coefficient, which depends on the friction between fluid and the wall of the orifice and the resistance force of orifice.

Substituting Eqs. (1), (2) into Eqs (3), (4) respectively, it is obtained as following

$$
\begin{aligned}
& \left(m_{0}+m_{1}\right) \ddot{x}+c_{s} \dot{x}+k_{s} x+A_{0}\left(A_{1} y\right) k=F \\
& m_{f} \ddot{y}+c_{f} \dot{y}=\left(A_{1} y\right) k
\end{aligned}
$$

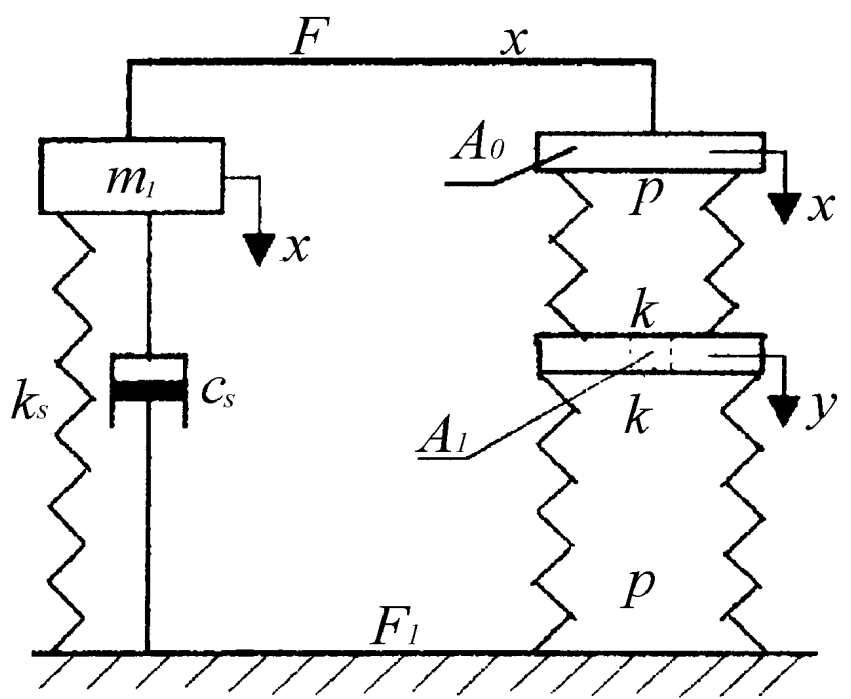

Fig. 2. Analytical model. 
according to Eq. (6), one yields

$$
A_{1} y=\frac{m_{f} \ddot{y}+c_{f} \dot{y}}{A_{1} k}
$$

substituting the Eq. (7) into Eq. (5), one has

$$
\left(m_{0}+m_{1}\right) \ddot{x}+c_{s} \dot{x}+k_{s} x+A_{0} \frac{m_{f} \ddot{y}+c_{f} \dot{y}}{A_{1}}=F
$$

according to the continuous condition (1) of fluid, one yields

$$
y=\frac{A_{0}}{A_{1}} x, \dot{y}=\frac{A_{0}}{A_{1}} \dot{x}, \ddot{y}=\frac{A_{0}}{A_{1}} \ddot{x}
$$

so, Eq. (8) can be rewritten as following

$$
\left(m_{0}+m_{1}\right) \ddot{x}+c_{s} \dot{x}+k_{s} x+\left[m_{f}\left(\frac{A_{0}}{A_{1}}\right)^{2} \ddot{x}+c_{f}\left(\frac{A_{0}}{A_{1}}\right)^{2} \dot{x}\right]=F
$$

or

$$
\left(m_{0}+m_{1}+m_{2}\right) \ddot{x}+\left(c_{s}+\tau A_{0}^{2}\right) \dot{x}+k_{s} x=F
$$

and

$$
m_{e}=\left(\frac{A_{0}}{A_{1}}\right)^{2} m_{f}, c_{e}=\left(\frac{A_{0}}{A_{1}}\right)^{2} c_{f}=\tau A_{0}^{2}
$$

where, $m_{e}$ and $c_{e}$ are the equivalent mass and the equivalent damp coefficient of the fluid flowing through orifice. According to the hydraulic theory, $c_{e}$ can be calculated by Eq. (11) [1]

$$
c_{e}=\tau A_{0}^{2} \quad(N \cdot \mathrm{s} / \mathrm{m})
$$

and

$$
\tau=\frac{128 v \rho h}{n \pi d^{4}} \quad\left(N \cdot \mathrm{s} / \mathrm{m}^{5}\right)
$$

where, $\tau$ is the resistance force coefficient of orifice; $v$ is the stick coefficient of fluid, and $v=1 \times 10^{-6}\left(\mathrm{~m}^{2} / \mathrm{S}\right)$ for water; $n$ denotes number of orifices; $d$ is the diameter of orifice; $\rho=1000\left(\mathrm{Kg} / \mathrm{m}^{3}\right)$

It is known that $m_{e}$ and $c_{e}$ depend on the ratio of the $A_{0}^{2} / A_{1}^{2}$. The behavior of vibration reduction can be improved by adjusting the ratio of $A_{0}^{2} / A_{1}^{2}$; the damp of HEM increases with increment of $A_{0}^{2} / A_{1}^{2}$. If the damp coefficient of the spring is ignored, the equation of motion (10) can be rewritten as following

$$
M \ddot{x}+\tau A_{0}^{2} \dot{x}+k_{s} x=F
$$

where, $M=m_{0}+m_{1}+m_{e}$.

\section{THE NATURAL VIBRATION CHARA- CTERISTICS AND DYNAMIC STIFFNESS OF HEM SYSTEM}

According to Eq. (13), the natural frequency of HEM without damp is

$$
\lambda_{0}=\sqrt{\frac{k_{s}}{M}}
$$

according to Eq. (13), the free vibration equation of HEM is

$$
\ddot{x}+2 \zeta \lambda_{0} \dot{x}+\lambda_{0}^{2} x=0
$$

and

$$
\zeta=\frac{\tau A_{0}^{2}}{2 M \lambda_{0}}
$$

The natural frequency of HEM with damp is

$$
\lambda_{d}=\lambda_{0} \sqrt{1-\zeta^{2}}
$$

It is assumed that the excitation is harmonic, that is,

$$
x=x_{0} e^{i \omega t}, F=F_{0} e^{i \omega t}
$$

where, $i=\sqrt{-1} ; \omega$ is the frequency of excitation displacement; $x_{0}$ denotes the displacement amplitude of excitation; $F_{0}$ indicates the amplitude of resultant forces.

Substituting Eq. (16) into Eq. (13), one yields

$$
-M \omega^{2} x_{0}+\tau A_{0}^{2} x_{0} i \omega+k_{s} x_{0}=F_{0}
$$

The dynamic stiffness $k_{d}(i \omega)$ of HEM is defined as $F_{0} / x_{0}$, or

$$
k_{d}(i \omega)=\frac{F_{0}}{x_{0}}=-M \omega^{2}+k_{s}+\tau A_{0}^{2} i \omega
$$

also, the amplitude of dynamic stiffness can be given by Eq. (19)

$$
\begin{aligned}
k_{d} & =\left|k_{d}(i \omega)\right| \\
& =\sqrt{M^{2} \omega^{2}+\left(-2 M k_{s}+\tau^{2} A_{0}^{4}\right) \omega^{2}+k_{s}^{2}} \\
& =k_{s} \sqrt{\left(1-\mu^{2}\right)^{2}+\eta^{2} \mu^{2}}
\end{aligned}
$$

where

$$
\mu=\frac{\omega}{\lambda_{0}}, \eta=\frac{\tau A_{0}^{2}}{M \lambda_{0}}=2 \zeta
$$

It is known from Eq. (19) that the dynamic stiff- 
ness increases with the increment of stiffness and the damp coefficient of HEM.

In order to show the effect of fluid inside orifice on the dynamic stiffness of HEM, only $m_{e}$ is considered in Eq. (19), one has

$$
\begin{aligned}
D(\alpha) & =|D(i \omega)| \\
& =\sqrt{m_{e}^{2} \omega^{4}+\left(-2 M k_{s}+\tau^{2} A_{0}^{4}\right) \omega^{2}+k_{s}^{2}} \\
& =k_{s} \sqrt{\left(1-\alpha^{2}\right)^{2}+\beta^{2} \alpha^{2}}
\end{aligned}
$$

where,

$$
\alpha=\frac{\omega}{\omega_{0}} ; \beta=\frac{\tau A_{0}^{2}}{m_{e} \omega_{0}} ; \omega_{0}=\sqrt{\frac{k_{s}}{m_{e}}}
$$

$D(\alpha)$ is called as the dynamic stiffness of equivalent fluid.

\section{TRANSFER FUNCTION OF FORCE} lowing

The amplitude of inputting force is given as fol-

$$
F_{0}=\left(-M \omega^{2}+\tau A_{0}^{2} i \omega+k_{s}\right) x_{0}
$$

the outputting force acting on the foundation is

$$
F_{1}=m_{e} \ddot{x}+\tau A_{0}^{2} \dot{x}+k_{s} x
$$

substituting Eq. (16) into Eq. (24), the amplitude outputting force is

$$
F_{10}=\left(-m_{e} \omega^{2}+\tau A_{0}^{2} i \omega+k_{s}\right) x_{0}
$$

the transfer function of force is

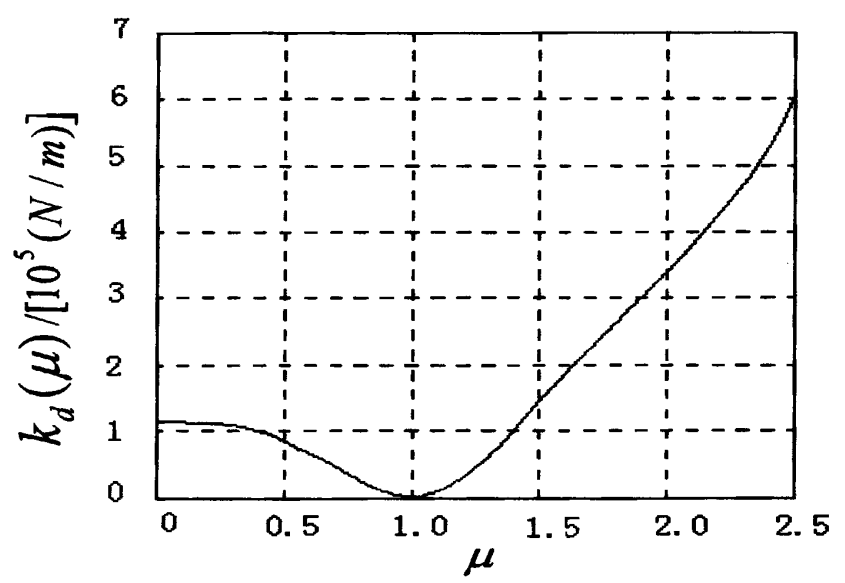

Fig. 3. Curve of HEM dynamic stiffness

$$
\begin{aligned}
T(i \omega) & =\frac{F_{10}}{F_{0}}=\frac{-m_{e} \omega^{2}+\tau A_{0}^{2} i \omega+k_{s}}{-M \omega^{2}+\tau A_{0}^{2} i \omega+k_{s}} \\
& =\frac{\left(1-\alpha^{2}\right)\left(1-\gamma \alpha^{2}\right)+\beta^{2} \alpha^{2}}{\left(1-\gamma \alpha^{2}\right)^{2}+\beta^{2} \alpha^{2}}+\frac{(1-\gamma) \beta \gamma^{3}}{\left(1-\gamma \alpha^{2}\right)^{2}+\beta^{2} \alpha^{2}} i \\
& =R_{e}+I_{m} i
\end{aligned}
$$

The amplitude of force transfer function is

$$
\begin{aligned}
& \mu(\alpha)=|T(i \omega)|=\sqrt{R_{e}^{2}+I_{m}^{2}} \\
& =\sqrt{\frac{\left[\left(1-\alpha^{2}\right)\left(1-\gamma \alpha^{2}\right)+\beta^{2} \alpha^{2}\right]^{2}+\left[(1-\gamma) \beta \alpha^{3}\right]^{2}}{\left[\left(1-\gamma \alpha^{2}\right)^{2}+\beta^{2} \alpha^{2}\right]^{2}}}
\end{aligned}
$$

where, $\alpha=\frac{\omega}{\omega_{0}} ; \gamma=\frac{M}{m_{e}}$.

\section{EXAMPLE}

The parameters of HEM shown in Fig. 1 are: $n=$ $43 ; d=4 \mathrm{~mm} ; h=21 \mathrm{~mm}$; the height of cylinder is $75 \mathrm{~mm}$.

According to the parameters, one has

$$
\begin{aligned}
& A_{0}=34.2 \mathrm{~cm}^{2} \\
& A_{1}=43 \times\left(\frac{0.3}{2}\right)^{2} \times 3.14=3.04 \mathrm{~cm}^{2} \\
& A_{0}^{2} / A_{1}^{2}=125.82 \\
& m_{f}=3.04 \times 10^{-4} \times 0.021 \times 1000=0.0064 \mathrm{~kg} \\
& m_{e}=125.82 \times 0.0064=0.8 \mathrm{~kg} \\
& m_{1}=0.957 \mathrm{~kg}, m_{0}=10 \mathrm{~kg}
\end{aligned}
$$

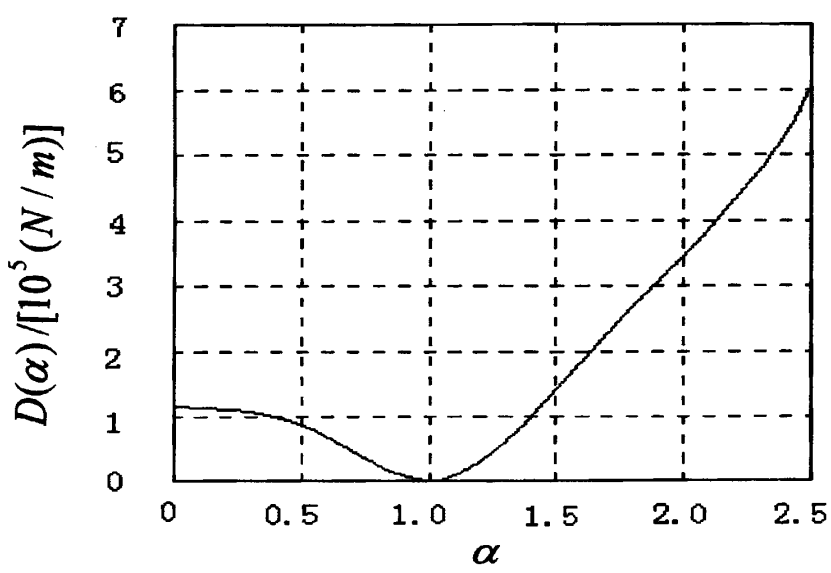

Fig. 4. Curve of fluid dynamic stiffness. 


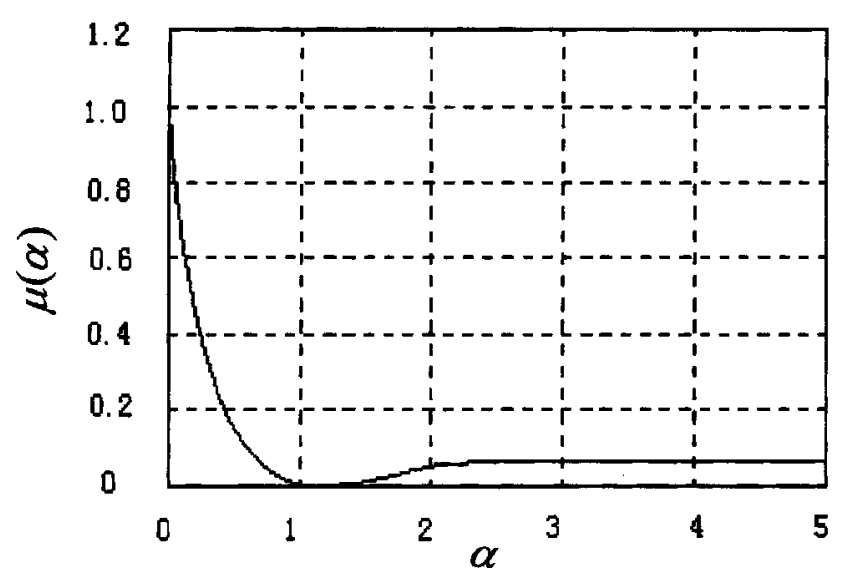

Fig. 5. Curve of force transfer function.

$$
M=m_{0}+m_{1}+m_{e}=11.76 \mathrm{~kg}
$$

The stiffness of spring on the bottom is calculated by Eq. (27) [9]

$$
k_{s}=\frac{G d^{4}}{8 D^{3} i}
$$

where, $G$ is the shear elasticity modulus, $G=1.68 \times 10^{11}$ $\left(\mathrm{N} / \mathrm{m}^{2}\right) ; d$ is the diameter of spring wire, $d=4 \mathrm{~mm} ; D$ denotes the diameter of $\operatorname{spring}, D=25 \mathrm{~mm} ; i$ is the curves of spring, $i=3$. The spring stiffness is $k_{s}=1.15$ $\times 10^{5}(\mathrm{~N} / \mathrm{m})$.

According to Eqs. (12), (14) and (22), one has

$$
\begin{aligned}
& \lambda_{0}=98.9(1 / \mathrm{s}), \omega_{0}=379(1 / \mathrm{s}), \\
& \tau=2.46 \times 10^{5}\left(\mathrm{~N} . \mathrm{s} / \mathrm{m}^{5}\right) .
\end{aligned}
$$

Substituting the above datum into Eq. (19), the curve of dynamic stiffness of HEM can be obtained and drawn in Fig. 3. Also, Fig. 4 shows the curve of dynamic stiffness of fluid inside the orifices. The curve of force transfer function is obtained according to Eq. (26) and described in Fig. 5.

It is known from Fig. 5 that the transfer function can be reduced effectively as $\alpha>1$ and $\mu>1$ corresponding to the higher frequency. Otherwise, the dynamic stiffness depends on the fluid damping and the force transfer function is less than 1 as $\alpha<1$ and $\mu<1$ corresponding to the lower frequency. So the HEM has high performance of vibration reduction in broad frequency region.

\section{CONCLUSIONS}

The metal hydraulic engine mount with orifice and two pistons is presented, and the analytical method of vibration reduction behavior is developed. The dynamic stiffness of HEM and the fluid oscillator are solved. Also, the force transfer function is determined. The analysis shows that the vibration response can be reduced effectively by using the metal hydraulic engine mount designed in this paper. The paper provides the novelty technology for reduction vibration of heavy vehicles.

\section{REFERENCES}

1. Frolov, K.V., Vibration in Technique, Machinary Press, Moscow (1995).

2. Gau, S.J. and Jeffry, D.C., "Experiment Study and Modeling of Hydraulic Mount and Engine System," Proceeding of the Noise and Vibration Conference of SAE, No. 951348, pp. 927-939 (1995).

3. Kazuto, S. and Katsuml, E., "Optimum Design Method for Hydraulic Engine Mounts," Proceeding of the Noise and Vibration Conference of SAE, No. 9110 55, pp. 463470 (1991).

4. Kim, G. and Singh, R., "A Study of Passive and Adaptive Hydraulic Engine Mount System with Emphasis on Nonlinear Characteristics," J. Sound Vib., Vol. 179, No. 3, pp. 427-453 (1995).

5. Qi, J.C., Li, R.X., Liu, Z.G., and Xu, X.X., "Study on Active Vibration Control for Ambulance Stretcher System Based on Sky-Hook Damper Theory," J. of Vib. Eng., No. 2, pp. 241-244 (1998). (in Chinese)

6. Takao, U., Kazuya, T., and Hiroshi, Ko., "High Performance Hydraulic Mount for Improving Vehicle Noise and Vibration," Proceeding of the Noise and Vibration Conference of SAE, No. 880073, pp. 450-458 (1988).

7. Thomas, J.R. and Singh, R., "Study of Nonlinear Hydraulic Engine Mounts Focusing on Decoupler Modeling and Design," Proceeding of the Noise and Vibration Conference of SAE, No. 971936, pp. 536-545 (1997).

8. Waliace, C.F., "Understanding Hydraulic Mounts for Improving Vehicle Noise, Vibration and Ride Qualities," Proceeding of the Noise and Vibration Conference of SAE, No. 850975, pp. 123-132 (1985).

9. Yang, L.M., Huang, K., Li, A.Z., and Cheng, S.X., Hand Book of Machine Design, National Defense Industry Press, Beijing (1988). 